\title{
1 Effects of fasting induced carbohydrate depletion on murine ischemic skeletal
}

2 muscle function.

3 Cameron A. Schmidt ${ }^{1,2}$, Emma J. Goldberg ${ }^{1,2}$, Tom D. Green ${ }^{1,2}$, Reema R. Karnekar ${ }^{1,2}$,

4 Jeffrey J. Brault ${ }^{1,3}$, Spencer G. Miller ${ }^{3}$, Adam J. Amorese ${ }^{1,2}$, Dean J. Yamaguchi, ${ }^{4,5}$, Espen

5 E. Spangenburg ${ }^{1,2}$, Joseph M. McClung ${ }^{1,2,4^{*}}$

7 1 Dept. of Physiology, Brody School of Medicine, East Carolina University, Greenville,

8 North Carolina, United States of America

9 2East Carolina Diabetes and Obesity Institute, East Carolina University, Greenville, North

10 Carolina, United States of America

$11{ }^{3}$ Dept. of Kinesiology, College of Health and Human Performance, East Carolina

12 University, Greenville, North Carolina, United States of America

13 4Department of Cardiovascular Sciences, East Carolina University, Greenville, North

14 Carolina, United States of America

15 5Division of Surgery, Brody School of Medicine, East Carolina University, Greenville, North

16 Carolina, United States of America

17

18 *Corresponding Author

19 Email: mcclungj@ecu.edu (J.M.M.)

20

21

22 


\section{Abstract}

24 Stored muscle carbohydrate supply and energetic efficiency constrain muscle functional

25 capacity during exercise and are influenced by common physiological variables (e.g. age,

26 diet, and physical activity level). Whether these constraints affect overall functional

27 capacity or the timing of muscle energetic failure during acute ischemia is not known. We

28 interrogated skeletal muscle contractile properties in two anatomically distinct hindlimb

29 muscles that have well characterized differences in energetic efficiency (locomotory-

30 extensor digitorum longus (EDL) and postural- soleus muscles) under conditions of

31 reduced carbohydrate supply. 180 mins of acute ischemia resulted in complete energetic

32 failure in all muscles tested, indicated by: loss of force production, substantial reductions

33 in total adenosine nucleotide pool intermediates, and increased adenosine nucleotide

34 degradation product - inosine monophosphate (IMP). These changes occurred in the

35 absence of apparent myofiber structural damage assessed histologically by both

36 transverse section and whole mount. Restriction of the available intracellular carbohydrate

37 pool by fasting ( $\sim 50 \%$ decrease in skeletal muscle) did not significantly alter the timing to

38 muscle functional impairment or affect the overall force/work capacities of either muscle

39 type. Fasting did cause rapid development of passive tension in both muscle types, which

40 may have implications for optimal timing of reperfusion or administration of precision

41 therapeutics. 


\section{Introduction}

50 Ischemic skeletal muscle necrosis occurs concurrently with several common clinical

51 conditions (e.g. peripheral arterial disease, compartment syndrome, or diabetic necrosis)

52 and is a complicating factor of successful muscle graft transplantation(1-3). The severity

53 of necrosis during an ischemic episode has long been considered a sole function of time,

54 temperature, and magnitude of the hypoxic insult $(4,5)$. However, the timing of the events

55 that precede irreversible functional impairment and necrosis during ischemia may also

56 depend on other key variables including: metabolic rate; contractile efficiency; and the size

57 of the stored carbohydrate pool(4). Carbohydrate metabolism is key, as muscle energy

58 supply becomes dependent on anaerobic fermentation of stored carbohydrate sources

59 during ischemia(6-8). Glycogen is the primary storage form of carbohydrate in skeletal

60 muscle, and its storage/utilization can be influenced by acute environmental factors as

61 well as chronic diseases(9-14).

62

63 Previous studies have examined the time dependent changes of metabolites and

64 contractile function in rodent skeletal muscle following ischemia with reperfusion (I/R)(15-

65 18). Several important observations can be gleaned from these studies: First, locomotory

66 (fast glycolytic) muscles experienced more damage compared to postural (slow oxidative)

67 muscles(15,17). Second, The degree of initial injury can have large effects on post

68 ischemic recovery time(16). Lastly, Optimal reperfusion timing is related to changes in

69 muscle metabolite levels during ischemia(18). A major limitation of $I / R$ studies is that it is

70 difficult to distinguish between the functional impairment and/or damage that is attributable

71 to the ischemia itself versus that caused by the reperfusion injury.

72

73 In a previous study, using an in vivo mouse hindlimb ischemia model (without reperfusion),

74 we found that myonecrosis develops between three and six hours after the onset of 
75 ischemia and is accompanied by a complete loss of contractile function(19). This led us

76 to examine the $<3$-hour time domain in this study to better characterize the exact temporal

77 nature of muscle functional impairments and metabolite changes that occur under

78 ischemic conditions. We hypothesized that reductions in stored muscle glycogen would

79 significantly shorten the amount of time that the muscles could remain functional during

80 ischemia. To test this hypothesis, we utilized fasting to induce an approximate $50 \%$

81 decrease in resting muscle glycogen and employed a carefully controlled experimental

82 system to assess the effects of carbohydrate depletion on isolated mouse hindlimb muscle

83 function during severe hypoxia and nutrient deprivation. Our data provide a novel

84 characterization of ischemic muscle mechanical/energetic failure and paint a detailed

85 picture of the timing of these impairments. This information will provide a valuable

86 resource to be used in conjunction with studies of ischemia/reperfusion in mouse hindlimb

87 ischemia models.

88

89 Materials and Methods

90

\section{Animals}

92 Adult male BALB/c mice ( $\mathrm{N}=32)$, aged $16-24$ weeks old, were obtained from Jackson

93 Laboratories (Bar Harbor, ME). All work was approved by the Institutional Animal Care

94 and Use Committee of East Carolina University. Animal care followed the Guide for the

95 Care and Use of Laboratory Animals, Institute of Laboratory Animal Resources,

96 Commission on Life Sciences, National Research Council. Washington: National

97 Academy Press, 1996. Animals had free access to water and food except during fasting

98 protocols, during which animals had free access to water only. 
101 For microvascular imaging, Dylight 594 conjugated Griffonia simplificolia isolectin $\mathrm{B}_{4}$ (GS-

$\left.102 \quad \mathrm{IB}_{4}\right)($ Vector Labs, Burlingame, $\mathrm{CA}$ ) was injected retro-orbitally one hour prior to sacrifice.

103 EDL and soleus muscles were dissected, and immersion fixed in 4\% paraformaldehyde.

104 BODIPY and DAPI staining was performed as previously described(20). Sarcomeric actin

105 staining was performed in PFA fixed whole mount muscles, following permabilization with

$10630 \mu \mathrm{g} / \mathrm{ml}$ saponin, using 200nM Alexa Fluor 488 conjugated phalloidin (Thermo Fisher,

107 Waltham MA). For $\mathrm{NAD}(\mathrm{P}) \mathrm{H}$ autofluorescence imaging, live muscle was dissected

108 following sacrifice and mounted at resting length. Live muscles were imaged in a glass

109 bottom dish in Krebs Ringer solution. All imaging was performed using an Olympus

110 FV1000 laser scanning confocal microscope (LSCM). Acquisition software was Olympus

111 FluoView FSW (V4.2). The objective used was 60X oil immersion (NA=1.35, Olympus

112 Plan Apochromat UPLSAPO60X(F)) or 30X (NA= 1.05, Olympus Plan Apochromat

113 UPLSAPO30XS). Images were $800 \times 800$ pixel with $2 \mu$ s/pixel dwell time. Detector noise

114 was reduced by application of a $3 \mathrm{X}$ line scanning kalman filter. Images were acquired in

115 sequential scan mode. $2 \mu \mathrm{M}$ DAPI was used for nuclear counterstaining (Sigma Aldrich,

116 St. Louis, MO) and was excited using the 405nm line of a multiline argon laser; emission

117 was filtered using a 490nm dichroic mirror and 430-470nm barrier filter. BODIPY and

118 AF488-phalloidin were excited using the 488nm line of a multiline argon laser; emission

119 was filtered using a 560nm dichroic mirror and 505-540nm barrier filter. Dylight 594 (GS-

$\left.120 \quad \mathrm{IB}_{4}\right)$ was excited using a $559 \mathrm{~nm}$ laser diode; emission was filtered using a $575-675 \mathrm{~nm}$

121 barrier filter. Zero detector offset was used for all images. The pinhole aperture diameter

122 was set to 105um (1 Airy disc). $\mathrm{NAD}(\mathrm{P}) \mathrm{H}$ autofluorescence has been shown to be highly

123 localized to skeletal muscle mitochondria(21). NAD(P)H autofluorescence was excited

124 using a mode locked pulsed laser (Mai Tai, Spectra Physics, Santa Clara, CA) tuned to

$125720 \mathrm{~nm}$. Emission was collected using separate non-descanned detectors. 


\section{Dystrophin/Laminin immunofluorescence in transverse muscle sections}

128 EDL and soleus muscles were embedded in optimal cutting temperature medium (OCT),

129 and frozen in liquid nitrogen cooled isopentane for cryosectioning. 10 $\mu \mathrm{m}$ sections were

130 cut using a CM-3050S cryostat (Leica, Wetzlar Germany) and collected on charged glass

131 slides. Sections were then fixed in $1: 1$ acetone/methanol for 10 minutes at $-20^{\circ} \mathrm{C}$,

132 rehydrated in $1 \mathrm{X}$ phosphate buffered saline (PBS), and blocked in $5 \%$ goat serum $+1 \mathrm{X}$

133 PBS for one hour at room temperature. Sections were then incubated with mouse anti-

134 human monoclonal dystrophin antibody (Thermo-Fisher, MA5-13526), and rabbit anti-rat

135 primary laminin antibody (Thermo-Fisher, A5-16287) at $4^{\circ} \mathrm{C}$ overnight. Sections were

136 washed $3 \mathrm{X}$ for 10 minutes with cold $1 \mathrm{X}$ PBS and incubated for 1 hour with Alexa-fluor 594

137 conjugated goat anti-rabbit IgG or Alexa-fluor 488 conjugated goat anti-mouse (highly

138 cross adsorbed) IgG2b secondary antibody (1:250, Invitrogen). Sections were mounted

139 using Vectashield hard mount medium without Dapi (Vector Labs). Images were taken

140 with an Evos FL auto microscope (Thermo Fisher, Waltham, MA) with a plan fluorite 20X

141 cover slip corrected objective lens (NA = 0.5, air). The following excitation/emission filter

142 cubes were used: GFP (470/22 nm Excitation; 510/42 nm Emission) and Texas Red

143 (585/29 nm Excitation; 624/40 nm Emission). 4X and 20X magnification images were

144 taken for each condition. Image processing was performed using ImageJ $(\mathrm{NIH}$, $145 \quad \mathrm{v} 1.51 \mathrm{f})(22)$

147 Fasting

148 Pilot testing was performed to determine the minimal fasting time to achieve an $\sim 50 \%$

149 reduction in resting skeletal muscle glycogen(23). Muscle glycogen reached the target

150 reduction after 24 hours of fasting (one dark cycle).

152 Measurement of muscle mechanical function 
153 Mice were sacrificed by cervical dislocation under isoflurane anesthesia (confirmed by

154 lack of pedal withdrawal reflex). Extensor digitorum longus (EDL) or soleus muscles were

155 carefully dissected and tied at both tendon ends with 5-0 silk sutures (Thermo Fisher,

156 Waltham, MA). Muscles were tied to an anchor at the proximal end and a dual mode force

157 transducer (Aurora 300B-LR, Aurora, ON, Canada) at the distal end in a vertical bath at

$15822^{\circ} \mathrm{C}$. All protocols were performed in the absence of additional carbon fuel sources (i.e.

159 amino acids, glucose, etc.) to restrict muscles to stored fuel supplies. The bath solution

160 was a modified Krebs Ringer solution described previously(24). All muscles were

161 dissected and mounted within 15 mins of sacrifice. Muscles were equilibrated in the bath

162 for 10 mins, and optimal length $\left(L_{0}\right)$ was determined by stimulating twitch contractions

163 (0.2ms pulse width, 1 pulse/train) at 10 second intervals and adjusting the length

164 incrementally until maximal force was achieved. Supramaximal stimulation voltage for

165 both muscle types was determined to be $20 \mathrm{~V} . \mathrm{L}_{\circ}(\mathrm{mm})$ was measured using a digital

166 microcaliper (Thermo Fisher, Waltham, MA). A force frequency curve was developed for

167 each muscle using stepwise increasing stimulation frequencies of $10,20,40,60,80,100$,

168 and $120 \mathrm{~Hz}$ (.2ms pulse width, pulses/train=half of the stim. Freq.). Baths were aerated

169 with $95 \% \mathrm{O}_{2} / 5 \% \mathrm{CO}_{2}$ (oxygenated; $\mathrm{O}_{2}$ condition) during $\mathrm{L}_{\mathrm{O}}$ determination and the initial

170 force frequency curve. The aeration source was then either left the same or changed to

$17195 \% \mathrm{~N}_{2} / 5 \% \mathrm{CO}_{2}$ (hypoxic; $\mathrm{N}_{2}$ condition) to simulate ischemia. The muscles were then

172 equilibrated for 10 mins, and an initial isokinetic contraction protocol was elicited in the $\mathrm{O}_{2}$

173 condition $(100 \mathrm{~Hz}$ isometric contraction for 0.8 seconds followed by a $3 \mathrm{~mm}$ shortening

174 phase over .3 seconds, then a return to $L_{\circ}$ over 30 s for the EDL; for the soleus $80 \mathrm{~Hz}$

175 isometric contraction was elicited for 0.8 seconds followed by a $4 \mathrm{~mm}$ shortening phase

176 over .4 seconds, then a return to $L_{o}$ over 30 s). The aeration source was then either left the

177 same or changed to $95 \% \mathrm{~N}_{2} / 5 \% \mathrm{CO}_{2}$ (hypoxic condition), with experimental conditions

178 alternated each time to reduce bias. The muscles were then equilibrated for 10 mins, 
179 followed by stimulated isokinetic contractions every 10 mins for 180 mins (18 total 180 contractions). We chose this timing based on our previous observation that excitation 181 contraction coupling is impaired in muscles isolated from BALB/c mice 180 minutes after 182 induction of acute hindlimb ischemia (in the absence of histological signs of tissue 183 necrosis)(19). A second force frequency curve was measured following the 180-min.

184 isokinetic protocol without changing the aeration source. Muscles were removed from the 185 apparatus, blot dried on paper, weighed, and flash frozen in liquid nitrogen for biochemical 186 analyses. Isometric time-tension integrals (TTI) were calculated by integrating over the 187 isometric (phase I) portion of the curve and are expressed in units of $\mathrm{N}^{*} \mathrm{~s} / \mathrm{cm}^{2}$. Isokinetic 188 work (W) was obtained by integrating the force over the length change during the 189 shortening (phase II) portion of the protocol and is expressed in units of $\mathrm{J} / \mathrm{cm}^{2}$.

191 Absolute isometric force measurements were normalized to mathematically approximated 192 cross-sectional areas of the muscles. The cross-sectional area for each muscle was 193 determined by dividing the mass of the muscle $(\mathrm{g})$ by the product of its optimal fiber length $194\left(L_{f}, \mathrm{~cm}\right)$ and estimated muscle density $\left(1.06 \mathrm{~g} \mathrm{~cm}^{-3}\right)$. Muscle force production was 195 expressed as specific force $\left(\mathrm{N} / \mathrm{cm}^{2}\right)$ determined by dividing the tension (N) by the 196 calculated muscle cross-sectional area. $L_{f}$ was obtained by multiplying $L_{o}$ by the standard 197 muscle length to fiber length ratio ( 0.45 for adult mouse EDL; 0.71 for soleus)(25). A gas 198 calibrated Clark electrode (Innovative instruments, Lake Park, NC) was used to assess 199 the oxygen saturation of the isolated bath medium under both aeration conditions prior to 200 carrying out the experiments. $\mathrm{O}_{2}$ conditions were approximately $90 \%$ saturation measured 201 at the center of the bath (after 10 mins of aeration). $\mathrm{N}_{2}$ conditions were $<2 \%$ saturation.

\section{Measurement of glycogen content in whole tissue}


204 Skeletal muscle and liver tissues were flash frozen in liquid nitrogen and stored at $-80^{\circ} \mathrm{C}$.

205 Glycogen assays were performed using acid hydrolysis and an enzyme coupled 206 assay(26). Briefly, tissue samples were digested/hydrolyzed under acidic conditions using $2072 \mathrm{~N}$ hydrochloric acid (Sigma Aldrich, St. Louis, MO) on a heating block at $95^{\circ} \mathrm{C}$ for 2 hours 208 with additional vortexing. Samples were neutralized with equal volume $2 \mathrm{~N}$ sodium

209 hydroxide (Sigma). A small amount of tris $\mathrm{HCl} \mathrm{pH} 7.0(\sim 1 \%$ of final volume) was added to 210 buffer the solution. Samples were added to a clear 96 well plate in duplicate and were 211 incubated with a solution containing: >2000U/L hexokinase (S. cerevisiae), >4000 U/L $212 \mathrm{NAD}^{+}$dependent glucose-6-phosphate dehydrogenase (L. mesenteroides), 4mM ATP, $2132 \mathrm{mM} \mathrm{Mg}^{2+}$, and $2 \mathrm{mM} \mathrm{NAD}^{+}$(Hexokinase reagent solution; Thermo Fisher). Water was 214 used in place of the reagent for background correction. A standard curve of D-glucose 215 (Sigma Aldrich) was used to calculate the concentrations of hydrolyzed glucosyl units in 216 each sample. Colorimetric measurement of $\mathrm{NAD}(\mathrm{P}) \mathrm{H}$ absorbance was made at 340nm 217 using a Cytation 5 microtiter plate reader (Biotek, Winooski, VT). Liver samples were 218 diluted 1:50 in water prior to enzyme coupled assays to obtain absorbance values within 219 the range of the standard curve. Data were normalized to tissue mass and represented 220 as nmoles glucose/mg tissue wet weight. The response coefficient $\left(R_{\text {Glyc }}\right)$ is defined as the 221 fractional change in experimental group mean relative to the basal group (i.e. Mean Basal 222 - Mean Experimental/Mean Basal $\left.{ }^{*} 100\right)$.

224 Ultra-performance liquid chromatography (UPLC) measurements of adenosine 225 nucleotides in whole tissue

226 UPLC measurements of adenosine nucleotides in whole muscle tissue have been 227 described in detail previously(27). Briefly, isolated muscles were flash frozen in liquid 228 nitrogen, homogenized in ice-cold perchloric acid using a glass on glass homogenizer, 229 and centrifuged to remove precipitated proteins. Samples were neutralized using 
230 potassium hydroxide and centrifuged a second time, to remove perchlorate salt.

231 Adenosine nucleotides and degradation products were assayed using an Acquity UPLC

$232 \mathrm{H}$ class system (Waters, Milford, MA). Metabolites were identified by comparison of peak

233 retention times of pure, commercially available standards (Sigma-Aldrich). These UPLC

234 measures can provide an index of intracellular energetic state. The amount of IMP reflects

235 longer periods of metabolic demand exceeding supply as the available adenylate pool is

236 decreased via irreversible deamination of AMP to IMP. Over the timeframe of these

237 stimulation protocols, IMP accumulation is a reliable measure of sustained mismatch

238 between ATP supply and demand. (Adenosine triphosphate-ATP, adenosine

239 diphosphate-ADP, adenosine monophosphate-AMP, and inosine monophosphate-IMP).

\section{Statistical Analysis}

242 Results of statistical comparisons are represented by means \pm standard error (SEM).

243 Sample variance (in figure panels) is represented by sample standard deviation (SD).

244 Analyses and plotting were carried out using Graphpad prism (V8.01; Windows 10).

245 Unpaired two-tailed t-tests were used for between group comparisons. For comparison of

246 means, $p$ values of $<0.05$ were considered statistically significant.

\section{Results}

249 Extensor digitorum longus (EDL) and soleus muscles were chosen for their known 250 differences in thermodynamic efficiency(28). The muscles also characteristically rely on 251 different modes of energy metabolism (glycolytic and oxidative metabolism 252 respectively)(29). The specialized nature of each muscle is highlighted for illustrative 253 purposes by whole mount imaging (Fig 1), contrasting the dramatically different 254 microvascular anatomy, cellular lipid droplet distribution (BODIPY), and mitochondrial 255 density/distribution $(\mathrm{NAD}(\mathrm{P}) \mathrm{H})$. 


\section{Fig 1: Microanatomy of Extensor digitorum longus (EDL) and soleus muscles differs}

257 in several key ways. Qualitative images highlighting a few of the key anatomical

258 differences between the fast twitch extensor digitorum longus (EDL) and slow twitch

259 soleus muscles (A). From left to right: (Left) Vessel density images are z-projections of

260 Dylight 594 conjugated lectin; (Middle) BODIPY images are z-projections of BODIPY

261 positive lipid droplets. Red signal in BODIPY images are lectin stained blood vessels. Blue

262 signal in BODIPY images are myonuclei. Arrows indicate BODIPY positive lipid droplets

263 in zoomed image inlays; (Right) Mitochondrial $\mathrm{NAD}(\mathrm{P}) \mathrm{H}$ images are optical sections of

264 reduced pyridine nucleotide autofluorescence in live isolated skeletal muscle. NAD(P)H

265 fluorescence intensity is mapped onto the image (highest intensity in white). Scale bars

266 are 25um.

268 Fasting is a well characterized and effective method of whole body carbohydrate depletion

269 in mice, due to their high thermal conductivity and large surface area to body volume

270 ratio(23). This method was chosen for this study because it is independent of the

271 confounding effects of exercise or contraction induced fatigue(30). The mean change in

272 bodyweight over the fasted period (24 hours) was $3.9 \pm 0.12$ grams, approximately $13 \%$

273 of the mean initial weight. We observed a large difference in stored glycogen levels

274 between fed and fasted groups in both liver ( 90\% lower) (Table 1) and skeletal muscle

275 ( $\sim 50 \%$ lower) (Table 1$)$. Interestingly, the resting glycogen concentration was higher in

276 the soleus than the EDL under both fed and fasted conditions. Additionally, soleus

277 muscles had a lower mean glycogen concentration in the fasted group relative to the fed

278 state (mean percent difference of $41.6 \%$ compared to $56.1 \%$ in the EDL groups; Table 1 ). 
281 Table 1: Basal tissue glycogen concentrations in the liver and skeletal muscle of fed Vs. fasted 282 groups.

\begin{tabular}{|l|l|l|l|l|}
\hline Tissue & Condition & $\begin{array}{l}\text { Glycogen } \\
(\mathbf{n m o l} / \mathbf{m g})\end{array}$ & $\begin{array}{l}\text { StDev } \\
(\mathbf{n m o l} / \mathbf{m g})\end{array}$ & \\
\hline Liver & Fed & $387.9^{*}$ & 88.43 & \\
\hline & Fasted & $42.0^{*}$ & 20.7 & 10.8 \\
\hline EDL & Fed & $34.4^{*} \#$ & 8.0 & 56.1 \\
\hline Soleus & Fasted & $19.3^{*} \#$ & 3.2 & \\
\hline & Fed & $61.9^{*} \#$ & 17.5 & 41.6 \\
\hline
\end{tabular}

283 Units are nanomoles hydrolyzed glucosyl units/milligram tissue wet weight (nmol/mg). * $\mathrm{p}<.05 \mathrm{Fed}$

284 V. Fasted Groups. \#p<.05 EDL V. Sol. N=4. Sample standard deviation (StDev).

286 Fasting had no effect on the isometric force-frequency relationship at baseline or under 287 any of the tested conditions in the EDL (Fig 2A) or soleus (Fig 2B), indicating reduced 288 carbohydrate pool size did not alter excitation-contraction coupling. Specific force values 289 for both muscles were consistent with those obtained previously(24). Additionally, we 290 observed characteristic reductions in maximal specific force following the $\mathrm{O}_{2}$ protocols 291 (and completely impaired force production following the $\mathrm{N}_{2}$ protocols) in both muscles (Fig 292 2A,B). Notably, the isometric force capacity during each protocol did not differ between 293 the fed and fasted groups in either the EDL (Fig 2C) or the soleus (Fig 2D). Similarly, the 294 work capacity over the course of the protocols did not differ for either muscle between the 295 fed and fasted states (Fig 2E,F). As expected, the force and work capacities were greatly 296 reduced under the $\mathrm{N}_{2}$ conditions compared to $\mathrm{O}_{2}$ conditions. 
298 Fig 2: Effects of carbohydrate depletion on excitation-contraction coupling and

299 force/work capacities. Specific force-frequency curves for EDL (A) and soleus (B). Basal

300 conditions are $95 \% \mathrm{O}_{2}$ prior to isokinetic protocol. (C,D) Specific force capacities were

301 obtained by summing the isometric portion of the time-tension integrals at each sampling

302 interval for the EDL and soleus respectively. (E,F) Specific work capacities were obtained

303 by summing the isovelocity (shortening) portion of the length-tension integrals at each

304 sampling interval for the EDL and soleus. $\mathrm{N}=8$ /treatment/group. Data are presented as

305 mean $\pm S D .{ }^{*} p<.05 \mathrm{O}_{2}$ Vs. $\mathrm{N}_{2}($ Vs. Basal $A, B)$.

308 Given that no substantial differences in force or work capacities were observed, we next

309 examined whether the timing of muscle functional impairments would differ between the

310 fed and fasted states. The time-tension integral (TTI) of the isometric portion of each

311 contraction was plotted as a function of the number of contractions (or time) during each

312 protocol for the EDL (Fig 3A) and soleus (Fig 3B). This measurement represents the

313 ability of the muscle to perform sustained non-shortening contractions. Additionally, the

314 length-time integral of the isokinetic portion of each contraction was also plotted against

315 the number of contractions for the EDL (Fig 3C) and soleus (Fig 3D). This measurement

316 represents the ability of the muscle to perform shortening work. Both sets of curves were

317 characterized by an inverse linear relationship under $\mathrm{O}_{2}$ conditions and a distinctly non-

318 linear inverse relationship under $\mathrm{N}_{2}$ conditions during the time and frequency domains of

319 the experiments. The muscles from the fasted group experienced more rapid reduction in

320 both TTI and work. All the tested muscles, however, experienced full impairment (defined

321 as force or work output $<10 \%$ of the initial value) within a relatively small $(\sim 10-20 \mathrm{~min}$.)

322 window of time. Passive tension was measured at the start of each contraction for the EDL

323 (Fig 3E) and soleus (Fig 3F). This measurement represents stiffening of the muscle, which 
324 may be due to several possible factors, including impaired calcium reuptake or cellular

325 swelling due to uncontrolled fluid uptake(31). None of the muscles experienced substantial

326 changes in passive tension during the $\mathrm{O}_{2}$ protocol. Large increases in passive tension

327 occurred in both muscles under $\mathrm{N}_{2}$ conditions. Interestingly, passive tension development

328 occurred earlier in the fasted groups (Fig 3E,F). To account for the possibility that the

329 muscles were accumulating excessive water, the wet weights of the EDL (Fig $\mathbf{3 G}$ ) and

330 soleus (Fig $\mathbf{3 H}$ ) were plotted. No differences in wet weight between the fed and fasted

331 states were observed in either muscle, and all the tested muscles accumulated additional

332 weight following the $\mathrm{N}_{2}$ protocol.

333

334 Fig 3: Effects of carbohydrate depletion on the timing of functional impairment

335 during ischemia. Isometric time-tension integrals (TTI) of each contraction over the 336 course of 18 contractions (or 180 minutes) under each condition for the EDL (A) and

337 soleus $(B)$. Isokinetic length-tension integrals (isokinetic work) of each contraction for the

338 EDL (C) and soleus (D). Developed passive tension (measured at the start of each

339 contraction) for the EDL (E) and soleus (F). Muscle wet weights obtained at the end of 340 each protocol for the EDL $(\mathrm{G})$ and soleus $(\mathrm{H}) . \mathrm{N}=8 /$ treatment/group. Data are presented 341 as mean \pm SD.

343 We next measured the muscle glycogen levels following the $\mathrm{O}_{2}$ and $\mathrm{N}_{2}$ protocols. The $\mathrm{N}_{2}$ 344 protocol reduced glycogen concentrations in all the muscles tested, relative to the $\mathrm{O}_{2}$ 345 condition (Table 2). Additionally, glycogen concentrations were lower in the fasted soleus 346 groups compared to the fed groups under both $\mathrm{O}_{2}$ and $\mathrm{N}_{2}$ conditions (Table 2). However, 347 glycogen concentrations did not differ between fed and fasted groups in the EDL muscles. 348 Using the response coefficient $\left(R_{\text {Glyc }}\right)$, allowed for comparison of each group mean to the 
349 basal values that are presented in Table 1. The patterns among both muscle types were

350 similar when represented this way. The largest differences observed were between $\mathrm{O}_{2}$

351 and $\mathrm{N}_{2}$ conditions and were not substantially different between fed and fasted groups.

352 Interestingly, the smallest difference in glycogen concentration observed was in the fasted

$353 \mathrm{O}_{2}$ condition for each muscle. This observation indicates the use of alternative (oxygen

354 dependent) fuel sources.

356 Table 2: Tissue glycogen concentrations in EDL and soleus muscles of fed V. fasted mice

357 following $\mathrm{O} 2$ or $\mathbf{N} 2$ protocols.

\begin{tabular}{|c|c|c|c|c|c|}
\hline Tissue & Group & Condition & Glycogen(nmol/mg) & StDev(nmol/mg) & $\mathbf{R}_{\text {Glyc }}(\%)$ \\
\hline \multirow[t]{4}{*}{ EDL } & Fed & $\mathrm{O}_{2}$ & $22.4 †$ & 4.4 & -34.8 \\
\hline & & $\mathrm{N}_{2}$ & $7.9 \dagger$ & 5.1 & -77.0 \\
\hline & Fasted & $\mathrm{O}_{2}$ & $18.8 \dagger$ & 7.3 & -2.5 \\
\hline & & $\mathrm{N}_{2}$ & $7.6 \dagger$ & 1.3 & -60.6 \\
\hline \multirow[t]{4}{*}{ Soleus } & Fed & $\mathrm{O}_{2}$ & $42.1 \dagger^{*}$ & 6.6 & -31.9 \\
\hline & & $\mathrm{N}_{2}$ & $29.0 \dagger^{*}$ & 1.6 & -53.1 \\
\hline & Fasted & $\mathrm{O}_{2}$ & $23.5 \dagger^{*}$ & 8.8 & -8.9 \\
\hline & & $\mathrm{N}_{2}$ & $9.8 \dagger^{*}$ & 4.2 & -62.0 \\
\hline
\end{tabular}

358 Units are nanomoles hydrolyzed glucosyl units/milligram tissue wet weight $(\mathrm{nmol} / \mathrm{mg})$. The

359 Response Coefficient $\left(\mathrm{R}_{\mathrm{Glyc}} \neg\right)$ indicates the percent change relative to the baseline group means 
360 (Presented in Table 1). Sample standard deviation (StDev). ${ }^{*} \mathrm{p}<.05$ Fed V. Fasted Groups. $\uparrow \mathrm{p}<.05$

361 O2 V. N2 Groups. N=4.

362

363 Total adenosine nucleotide (TAN) concentrations were examined as a measure of the 364 aggregate tissue energetic state at the end of each protocol. Reductions in the 365 concentrations of the total adenosine nucleotide pool, and accumulation of IMP, are 366 measures of the muscles' inability to resynthesize ATP(27). Following the $\mathrm{O}_{2}$ protocol, the

367 TAN pool decreased slightly in both EDL (Fig 4C) and soleus (Fig 4D) muscles compared 368 to our reported baseline values (Fig 4 A,B), but did not differ between fed and fasted 369 groups. Following $\mathrm{N}_{2}$ protocols, there were large decreases in the TAN pool in both the 370 EDL (Fig 4E) and soleus (Fig 4F), with accompanying increases in the tissue IMP 371 concentrations (Fig 4G,H). However, no substantial differences were observed between 372 the fed and fasted groups for either muscle type.

374 Fig 4: Metabolic characteristics of isolated EDL and soleus muscles under limiting 375 conditions of resting glycogen and oxygen availability. Total adenosine nucleotide 376 concentrations at baseline for the EDL $(A)$ and soleus $(B)$. TAN concentrations following 377 the $180 \mathrm{~min}$. protocol in $95 \% \mathrm{O}_{2}$ for the EDL (C) and soleus (D). TAN concentrations 378 following the $180 \mathrm{~min}$. protocol in $95 \% \mathrm{~N}_{2}(\mathrm{E}, \mathrm{F})$. IMP accumulation after each protocol, 379 compared across all three conditions for the EDL $(G)$ and soleus $(H)$. $380 \mathrm{~N}=4 /$ treatment/group. Data are presented as mean $\pm \mathrm{SD} .{ }^{*} \mathrm{p} \leq .05 \mathrm{O} 2 \mathrm{~V}$. N2 (V. Basal) 381 Groups.

383 Previous reports have indicated that dystrophin IF staining is rapidly reduced in skeletal 384 and cardiac muscle during early myonecrosis(19,32). Immunofluorescent staining for the 
385 sarcolemmal protein dystrophin and the extracellular matrix protein laminin was performed

386 on a subset of transverse sectioned muscles to assess the possibility that muscles were

387 incurring damage during the contraction protocols. No apparent changes were observed

388 in the EDL (Fig $\mathbf{5 A}$ ) or soleus (Fig $\mathbf{5 C}$ ) under $\mathrm{O}_{2}$ or $\mathrm{N}_{2}$ conditions, indicating that the muscle

389 tissue remained intact during the experiments. Degradation of myofibrillar structures are

390 another well characterized indicator of myonecrosis development(33). Parallel

391 assessments were made to accompany the dystrophin/laminin stain. Fibrous actin was

392 stained in fixed whole mount muscle specimens utilizing optical sectioning to assess the

393 intramyofibrillar-IMF and perinuclear-PN regions of the myofibers at baseline and following

394 the $\mathrm{N}_{2}$ protocol in the EDL (Fig 5B) and soleus (Fig 5D). Together these assessments did

395 not reveal any qualitative indication of damage.

397 Fig 5: Assessment of structural integrity of the muscles following experimental

398 protocols. To control for the possibility that the muscles were structurally damaged during

399 the contraction protocols, we performed immunofluorescence against sarcolemmal and

400 extracellular matrix proteins. Image panels of dystrophin (green), and laminin (red) stained

401 transverse EDL (A) and soleus (C) muscle sections under each of the conditions tested.

402 Sarcomeric actin was stained using phalloidin (Cyan) in fixed/permeabilized whole mount

403 muscles at baseline or following 180 mins of severe hypoxia $\left(95 \% N_{2}\right)$; EDL (B) and soleus

404 (D). Optical sectioning facilitated imaging in the intra-myofibrillar (IMF) and perinuclear

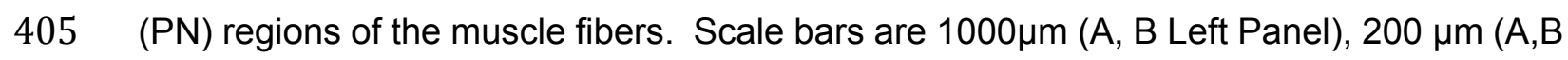
406 right panels), and $25 \mu \mathrm{m}(\mathrm{B}, \mathrm{D})$.

\section{Discussion}


410 Skeletal muscle is among the most metabolically dynamic tissues in the body, and is

411 capable of sustaining a 100-fold change in ATP utilization rate during contraction(34). The

412 total cost of ATP during contraction is proportional to the duration, intensity, and type (i.e.

413 shortening vs. non-shortening)(35). Glycogen is the primary storage form of glucose in

414 skeletal muscle, and is a major source of fuel during most forms of muscle activity(36).

415 Importantly, glycogen is also the primary source of stored fuel utilized to regenerate ATP

416 via substrate level phosphorylation in anaerobic glycolysis during severe hypoxia(35).

417 Depletion of stored muscle glycogen by fasting or exhaustive exercise results in impaired

418 fatigue resistance and recovery in isolated rodent muscles under normoxic conditions(36-

419 38). Under ischemic conditions, this effect would be expected to lead to cumulative

420 reductions in energetic capacity due to the inability to resynthesize ATP and phospho-

421 creatine $(\mathrm{PCr})$ that is used in support of contraction or resting metabolic processes.

422 Overnight fasting in rodents results in more dramatic metabolic effects than human

423 overnight fasting, but induces experimentally reproduceable reductions in systemic

424 carbohydrate stores that are similar to more extreme physiological conditions such as

425 hyperinsulinemia, hypoglycemia, or post exercise recovery $(11,14,23,39)$. We were

426 somewhat surprised to find that dramatically reduced muscle glycogen levels had no

427 substantial effect on the timing or magnitude of muscle functional impairment under

428 ischemic conditions. Our findings indicate that both muscle types retain a large pool of

429 stored glycogen that is non-essential for reserve mechanical force production during

430 hypoxia. It is not clear what the reserve glycogen pool contributes to in vivo during fasting.

431 Future studies could be directed to investigate its' potential involvement in the

432 maintenance of systemic glucose homeostasis through the production of free amino acids

433 (i.e. alanine and glutamine) or lactate which can be converted to glucose in the

434 liver(40,41). 
436 In mouse EDL and soleus muscles, as much as $50 \%$ of the resting metabolic rate has

437 been attributed to maintenance of intracellular calcium homeostasis(42). We observed a

438 rapid increase in passive tension development in the fasted group relative to the fed group

439 under $\mathrm{N}_{2}$ conditions in both muscle types. The observed increase in the rate of passive

440 tension development was the only measurement that was substantially different between

441 the fed and fasted groups. This phenomenon is most likely indicative of progressive

442 impairment of calcium handling as the capacity for ATP re-synthesis was gradually

443 depleted(27). This effect may have implications for reperfusion timing, as it has been noted

444 that calcium handling impairment prior to reperfusion is associated with poor salvage

445 outcomes $(4,43)$.

446 The dynamic requirements of ATP during muscle contraction require a similarly dynamic

447 supply of carbon fuel sources that are derived from both blood and intracellular stores.

448 Physiological and anatomical adaptations (i.e. capillary density, size of stored energy

449 substrate pools, and mitochondrial density/function) are known to facilitate large

450 differences in capacity for spontaneous vs. sustained exercise in different species(44).

451 Similar adaptive differences are highlighted in the distinct microanatomical differences of

452 mouse locomotory EDL and postural soleus muscles. These adaptive differences,

453 combined with their similar size and relatively homogeneous fiber type compositions,

454 make these muscles excellent candidates for comparative studies of muscle energy

455 metabolism.

456 Skeletal muscle fiber types are categorized by a range of intrinsic metabolic and 457 mechanical properties(45). Human muscles are generally of mixed fiber type, but mouse 458 muscles consist of more homogeneous fiber type distributions, making them a practical 459 model for studying fiber type specific effects (soleus: 1:1 slow type I/fast type Ila; EDL: 9:1

460 fast type IIb/fast type Ila)(29,46). At face value, it may seem intuitive that fast glycolytic 
461 fiber types would be better suited to performance during hypoxia due to their preference

462 for stored carbohydrate dependent energy metabolism $(36,37,47)$. However, several

463 studies have indicated a high degree of sensitivity of fast glycolytic muscles to

464 ischemia/reperfusion injury $(16,17,48)$. One important contributing factor to this effect is an

465 energetic inefficiency of contraction due to interactions at the level of the acto-myosin

466 crossbridges $(28,49)$. In the present study, we observed that Soleus muscles stored more

467 glycogen at baseline, had greater specific force/work capacities, and produced absolute

468 force for a longer period during ischemia compared to EDL muscles. These observations

469 are consistent with previous reports $(48,50)$. Though the absolute differences in glycogen

470 concentrations between groups were larger in the soleus compared to the EDL, the

471 response coefficient $\left(R_{\text {Glyc }}\right)$ which facilitates interpretation of group differences relative to

472 their baseline concentration, indicated that the patterns of utilization were not different

473 between the two types of muscles. We interpret these findings to mean that the greater

474 basal glycogen concentration observed in the soleus muscles was likely not the primary

475 factor underlying it's enhanced ischemic mechanical performance.

\section{Conclusion}

478 Investigating the key factors that affect the timing of muscle energetic failure during 479 ischemia will aid in identifying optimal windows for therapeutic intervention. We predicted

480 that the amount of stored carbohydrate is one such factor, as it is a major contributor to 481 anaerobic energy metabolism and is influenced by several physiologically relevant 482 conditions. We conclude that mouse hindlimb muscles maintain a large pool of stored 483 carbohydrate that is utilized during fasting but does not contribute substantially to the

484 timing of functional decline during acute ischemia. The carbohydrate lowering effects of 485 fasting did not substantially affect the total capacity or timing of contractile function 486 impairment in either muscle type. However, fasting did result in substantial increases in 
487 early passive tension development, which may have implications for the timing of

488 reperfusion or therapeutic administration. We also found that soleus muscles maintained

489 a greater total force capacity and became impaired more slowly than EDL muscles,

490 independent of glycogen utilization during the experimental period. This finding supports

491 several previous observations and bolsters the notion that susceptibility to acute ischemic

492 injury is not uniform across muscle types.

493

494 References:

495 1. Liu J, Saul D, Böker KO, Ernst J, Lehman W, Schilling AF. Current Methods for

496 Skeletal Muscle Tissue Repair and Regeneration. Biomed Res Int. 2018;2018:1-

$497 \quad 11$.

498 2. Dolan NC, Liu K, Criqui Michael H, Greenland P, Guralnik Jack M, Chan C, et al.

499 Peripheral artery disease, diabetes, and reduced lower extremity functioning.

$500 \quad$ Diabetes Care. 2002;25(1):113-20.

501 3. Callum K, Bradbury A. ABC of arterial and venous disease: Acute limb ischaemia.

$502 \quad$ BMJ. 2000;320(7237):764-7.

503 4. Paradis S, Charles A-L, Meyer A, Lejay A, Scholey JW, Chakfé N, et al.

504 Chronology of mitochondrial and cellular events during skeletal muscle ischemia-

505 reperfusion. Am J Physiol - Cell Physiol. 2016;310(11):C968-82.

506 5. Petrasek PF, Homer-Vanniasinkam S, Walker PM. Determinants of ischemic

507 injury to skeletal muscle. J Vasc Surg. 1994;19(4):623-31.

508 6. Jansson E, Johansson J, Sylven C, Kaijser L. Calf muscle adaptation in

509 intermittent claudication. Side-differences in muscle metabolic characteristics in

510 patients with unilateral arterial disease. Clin Physiol. 1988;8(1):17-29.

511 7. Theodore Kalogeris, Christopher P. Baines, Maike Krenz RJK. Cell Biology of

512 Ischemia/Reperfusion Injury. Int Rev Cell Mol Biol. 2012;298:229-317. 
513 8. Spriet LL, Soderlund K, Bergstrom M, Hultman E. Anaerobic energy release in

$514 \quad$ skeletal muscle during electrical stimulation in men. J Appl Physiol.

$515 \quad 1987 ; 62(2): 611-5$.

516 9. Damsbo P, Vaag A, Hother-Nielsen O, Beck-Nielsen H. Reduced glycogen

517 synthase activity in skeletal muscle from obese patients with and without Type 2

518 (non-insulin-dependent) diabetes mellitus. Diabetologia. 1991;34(4):239-45.

519 10. Bergstrom J, Hermansen L, Hultman E, Saltin B. Diet, Muscle Glycogen and

$520 \quad$ Physical Performance. Acta Physiol Scandanavia. 1967;(71):140-50.

521 11. Vollestad NK, Blom C. Effect of varying intensity on glyocgen depletion in human

522 muscle fibres. Acta Physiol Scand. 1985;125:395-405.

523 12. Askew CD, Green S, Walker PJ, Kerr GK, Green AA, Williams AD, et al. Skeletal

524 muscle phenotype is associated with exercise tolerance in patients with peripheral

525 arterial disease. J Vasc Surg. 2005;41(5):802-7.

526 13. McGuigan MR, Bronks R, Newton RU, Sharman MJ, Graham JC, Cody D V, et al.

$527 \quad$ Muscle fiber characteristics in patients with peripheral arterial disease. Med Sci

$528 \quad$ Sports Exerc. 2001;33(12):2016-21.

529 14. Cohen N, Rossetti L, Shlimovich P, Halberstam M, Hu M, Shamoon H.

530 Counterregulation of hypoglycemia: Skeletal muscle glycogen metabolism during

531 three hours of physiological hyperinsulinemia in humans. Diabetes.

$532 \quad 1995 ; 44(4): 423-30$.

533 15. Woitaske MD, McCarter RJ. Effects of fiber type on ischemia-reperfusion injury in

534 mouse skeletal muscle. Vol. 102, Plastic and reconstructive surgery. 1998. p.

535 2052-63.

536 16. Vignaud A, Hourde C, Medja F, Agbulut O, Butler-Browne G, Ferry A. Impaired

537 skeletal muscle repair after ischemia-reperfusion injury in mice. J Biomed

$538 \quad$ Biotechnol. 2010;2010. 
539 17. Chan RK, Austen WG, Ibrahim S, Ding GY, Verna N, Hechtman HB, et al.

$540 \quad$ Reperfusion injury to skeletal muscle affects primarily type II muscle fibers. J Surg

$541 \quad$ Res. 2004;122(1):54-60.

542 18. Idstrom J, Soussi B, Elander A, Bylund-Fellenius A. Purine metabolism after in

543 vivo ischemia and reperfusion in rat skeletal muscle. Am Physiol Soc.

$544 \quad$ 1990;258(6):1668-73.

545 19. Schmidt CA, Amorese AJ, Ryan TE, Goldberg EJ, Tarpey MD, Green TD, et al.

546 Strain-Dependent Variation in Acute Ischemic Muscle Injury. Am J Pathol. 2018

$547 \quad$ May $1 ; 188(5): 1246-62$.

548 20. Spangenburg EE, Pratt SJP, Wohlers LM, Lovering RM. Use of BODIPY

$549 \quad(493 / 503)$ to visualize intramuscular lipid droplets in skeletal muscle. J Biomed

$550 \quad$ Biotechnol. 2011;

551 21. Rothstein EC, Carroll S, Combs CA, Jobsis PD, Balaban RS. Skeletal muscle

$552 \quad \mathrm{NAD}(\mathrm{P}) \mathrm{H}$ two-photon fluorescence microscopy in vivo: Topology and optical inner

553 filters. Biophys J. 2005;88(3):2165-76.

554 22. Schneider CA, Rasband WS, Eliceiri KW. NIH Image to ImageJ: 25 years of

555 image analysis. Nat Methods. 2012 Jun;9(7):671-5.

556 23. Jensen TL, Kiersgaard MK, Sørensen DB, Mikkelsen LF. Fasting of mice: A

557 review. Lab Anim. 2013;47(4):225-40.

558 24. Tarpey MD, Amorese AJ, Balestrieri NP, Ryan TE, Schmidt CA, McClung JM, et

559 al. Characterization and utilization of the flexor digitorum brevis for assessing

560 skeletal muscle function. Skelet Muscle. 2018;8(1):1-15.

561 25. Barton ER, Lynch G, Khurana TS, Grange RW, Raymackers J-M, Dorchies O, et

562 al. Measuring isometric force of isolated mouse muscles in vitro. Exp Protoc DMD

$563 \quad$ Anim Model Treat-NMD Neuromuscul Network. 2008;1(2):14.

564 26. Passonneau J V, Lauderdale VR. A Comparison of Three Methods of Glycogen 
565 Measurement in Tissues. Anal Biochem. 1974;60(2):405-12.

566 27. Brault JJ, Pizzimenti NM, Dentel JN, Wiseman RW. Selective inhibition of ATPase

567 activity during contraction alters the activation of p38 MAP kinase isoforms in

568 skeletal muscle. J Cell Biochem. 2013;114(6):1445-55.

569 28. Barclay CJ. The basis of differences in thermodynamic efficiency among skeletal

570 muscles. Clin Exp Pharmacol Physiol. 2017;44(June):1279-86.

571 29. Augusto V, Padovani CR, Eduardo G, Campos R. Skeletal Muscle Fiber Types in 572 C57BI6J Mice. Braz J Morphol Sci. 2004;21(2):89-94.

573 30. Jennische E. Ischaemia Induced Injury in Glycogen-Depleted Skeletal Muscle.

574 Selective Vulnerability of FG Fibres. Acta Physiol Scandanavia. 1985;125:727-34.

575 31. Law DJ. Myofibrils Bear Most of the Resting Tension in Frog Skeletal Muscle.

$576 \quad$ Science (80- ). 1985;230:1280-2.

577 32. Armstrong SC, Latham CA, Shivell CL, Ganote CE. Ischemic Loss of

578 Sarcolemmal Dystrophin and Spectrin: Correlation with Myocardial Injury. J Mol

$579 \quad$ Cell Cardiol. 2001;33(6):1165-79.

580 33. Fielding RA, Manfredi TJ, Ding W, Fiatarone MA, Evans WJ, Cannon JG. Acute

581 phase response in exercise. III. Neutrophil and IL-1 beta accumulation in skeletal

582 muscle. Am Physiol Soc. 1993;265(1):166-72.

583 34. Weibel ER. Exercise-induced maximal metabolic rate scales with muscle aerobic $584 \quad$ capacity. J Exp Biol. 2005;208(9):1635-44.

585 35. Barclay CJ. Energetics of contraction. Compr Physiol. 2015;5(2):961-95.

586 36. Allen DG, Lamb GD, Westerblad H. Skeletal Muscle Fatigue : Cellular

587 Mechanisms. Physiol Rev. 2008;88:287-332.

588 37. Chin ER, Allen DG. Effects of reduced muscle glycogen concentration on force, 589 Ca2+release and contractile protein function in intact mouse skeletal muscle. J

$590 \quad$ Physiol. 1997;498(1):17-29. 
591 38. Ørtenblad N, Westerblad H, Nielsen J. Muscle glycogen stores and fatigue. J

$592 \quad$ Physiol. 2013;591(18):4405-13.

593 39. Adeva-Andany MM, González-Lucán M, Donapetry-García C, Fernández-

594 Fernández C, Ameneiros-Rodríguez E. Glycogen metabolism in humans. BBA

$595 \quad$ Clin. 2016;5:85-100.

596 40. Garber A, Karl I, Kipnis D. Alanine and Glutamine Synthesis and Release from 597 Skeletal Muscle I. J Biol Chem. 1976;251(3):836-43.

598 41. Garber A, Karl I, Kipnis D. Alanine and Glutamine Synthesis and Release from 599 Skeletal Muscle II. J Biol Chem. 1976;251(3):836-43.

600 42. Smith IC, Bombardier E, Vigna C, Tupling AR. ATP Consumption by 601 Sarcoplasmic Reticulum Ca2+ Pumps Accounts for $40-50 \%$ of Resting Metabolic

43. Walker PM. Ischemia/Reperfusion Injury in Skeletal Muscle. Ann Vasc Surg. $1991 ; 5(4): 399-402$.

44. Weibel ER, Taylor CR, Weber JM, Vock R, Roberts TJ, Hoppeler H. Design of the oxygen and substrate pathways. VII. Different structural limits for oxygen and substrate supply to muscle mitochondria. J Exp Biol. 1996;199:1699-709.

608 45. Saltin B, Gollnick PD. Skeletal Muscle Adaptability: Significance for Metabolism and Performance. Skelet Muscle. 1983;10:555-631.

610 46. Simoneau J, Bouchard C. Human variation in skeletal muscle fiber-type proportion 611 and enzyme activities. Am J Physiol .... 1989;257(4):567-72.

612 47. Westerblad H, Allen DG, Bruton JD, Andrade FH, Lännergren J. Mechanisms 613 underlying the reduction of isometric force in skeletal muscle fatigue. Acta Physiol $614 \quad$ Scand. 1998;162(3):253-60.

615 48. Howlett RA, Hogan MC. Effect of hypoxia on fatigue development in rat muscle 616 composed of different fibre types. Exp Physiol. 2007;92(5):887-94. 
617 49. Barclay CJ, Constable JK, Gibbs CL. Energetics of fast- and slow- twitch muscles

618 of the mouse. J Physiol. 1993;472:61-80.

619 50. Bonen A, Mcdermott J, Tan M. Glycogenesis and Glyconeogenesis in Skeletal:

$620 \quad$ Effects of $\mathrm{pH}$ and Hormones. Am J Physiol - Endocrinol Metab. 1990;258(4):693-

621700.

622

623

624

625

626

627

628

629

630

631

632

633

634

635

636

637

638

639

640

641

642 
bioRxiv preprint doi: https://doi.org/10.1101/846774; this version posted November 18,2019 . The copyright holder for this preprint (which was not certified by peer review) is the author/funder, who has granted bioRxiv a license to display the preprint in perpetuity. It is made available under aCC-BY 4.0 International license.

643

644

645

646

647

648 


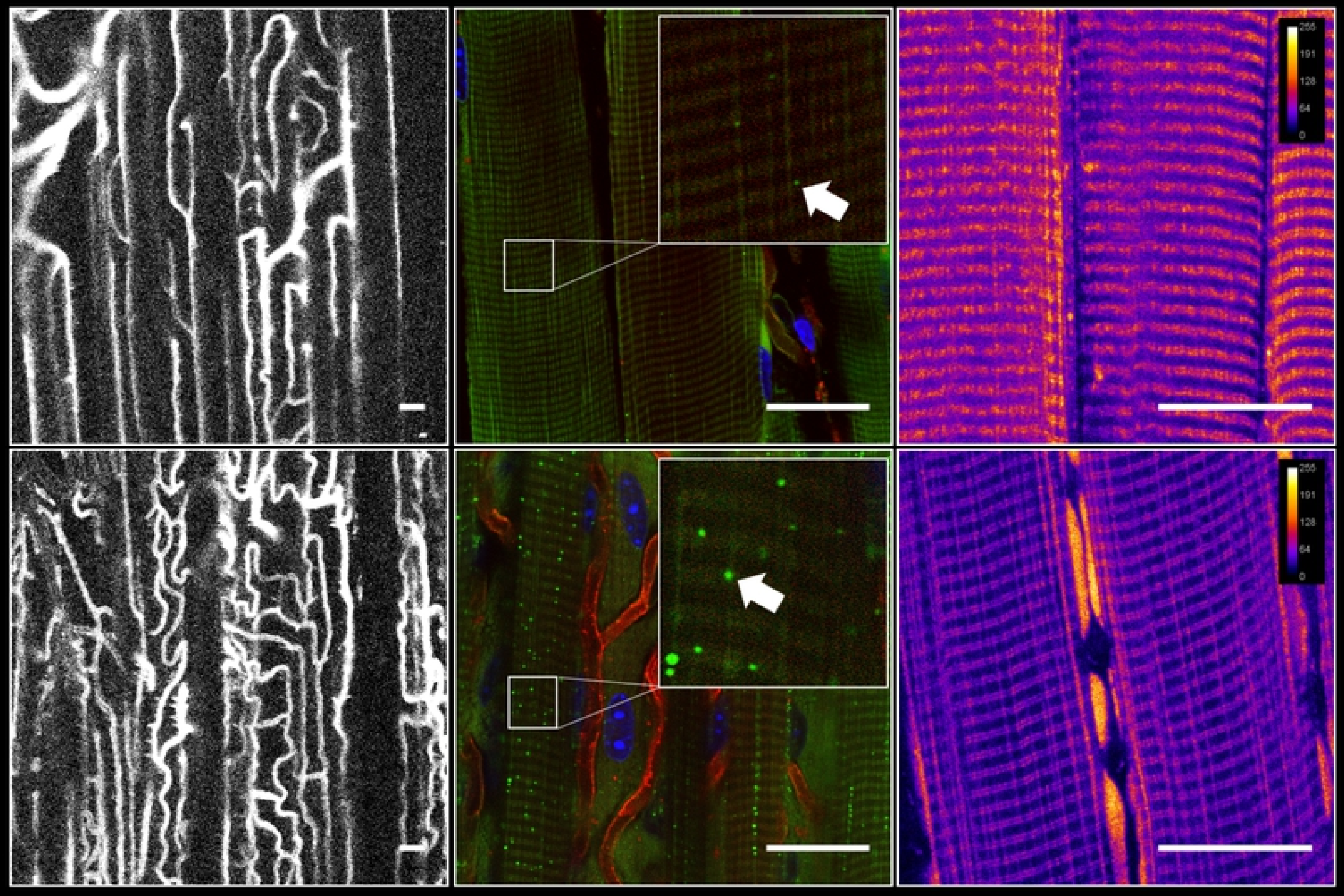

Figure 1 


\section{EDL}

\section{Soleus}
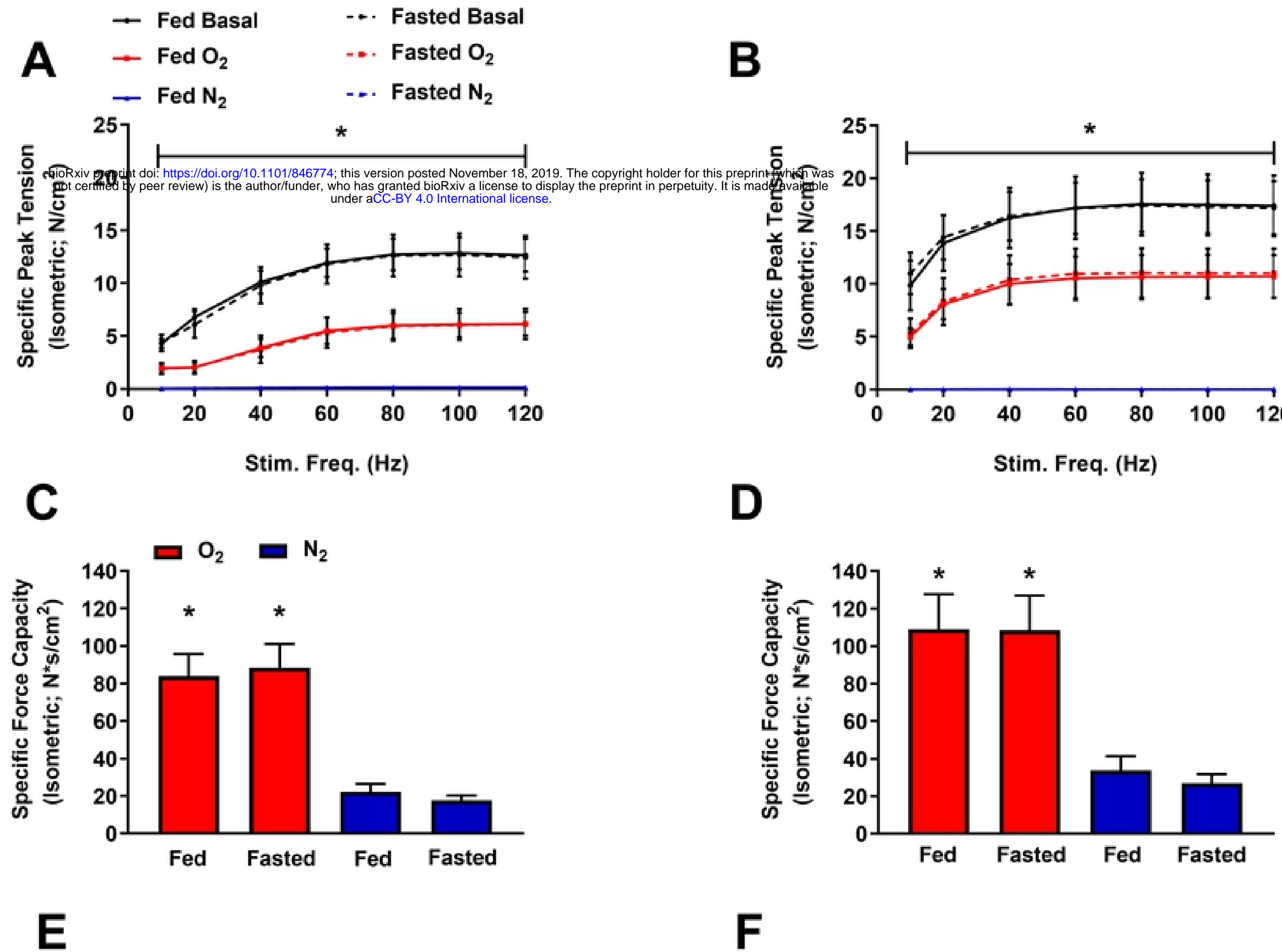

D
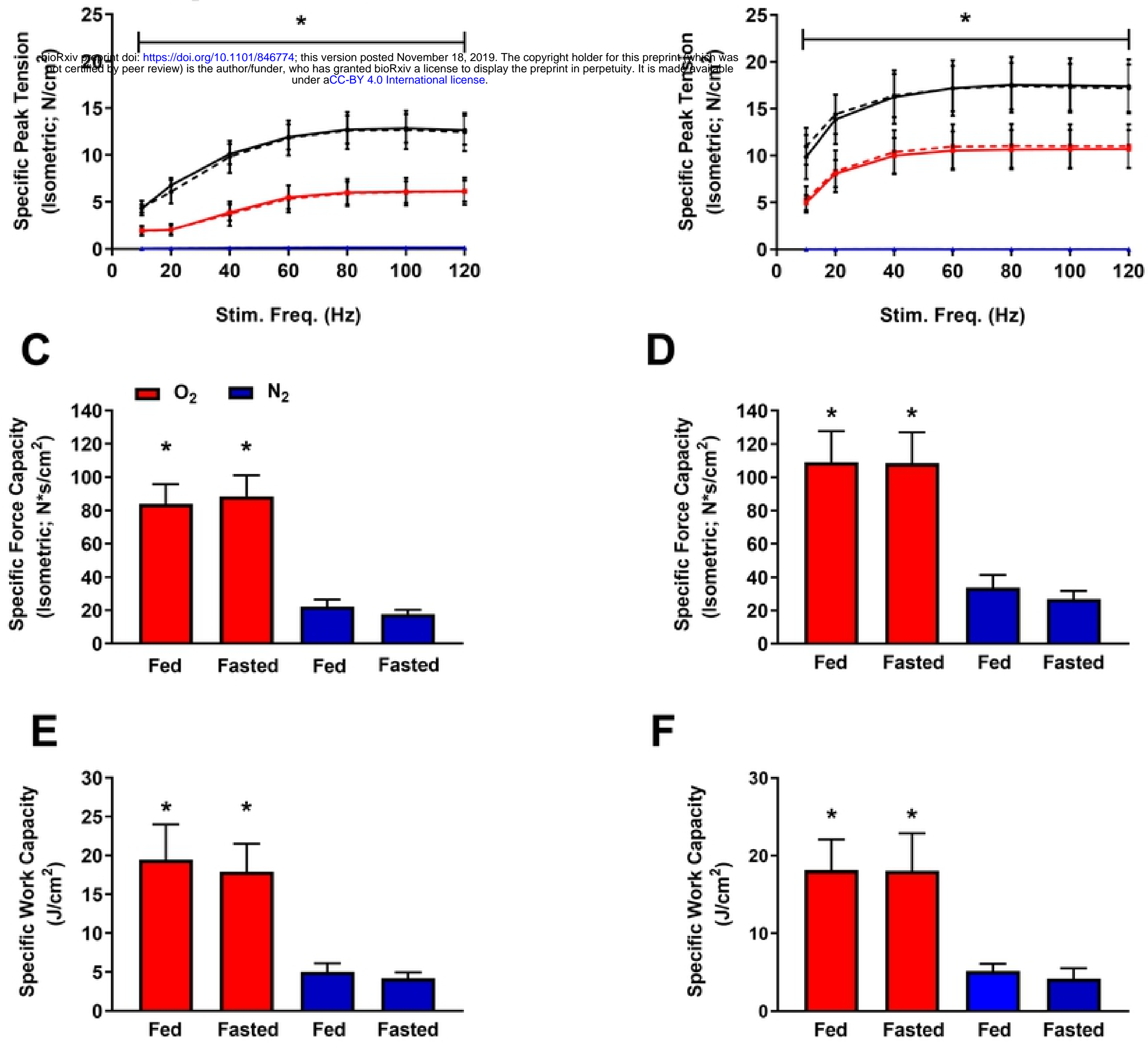

Figure 2 
A $\quad \rightarrow$ Fed $\mathrm{O}_{2} \rightarrow$ Fasted $\mathrm{O}_{2}$

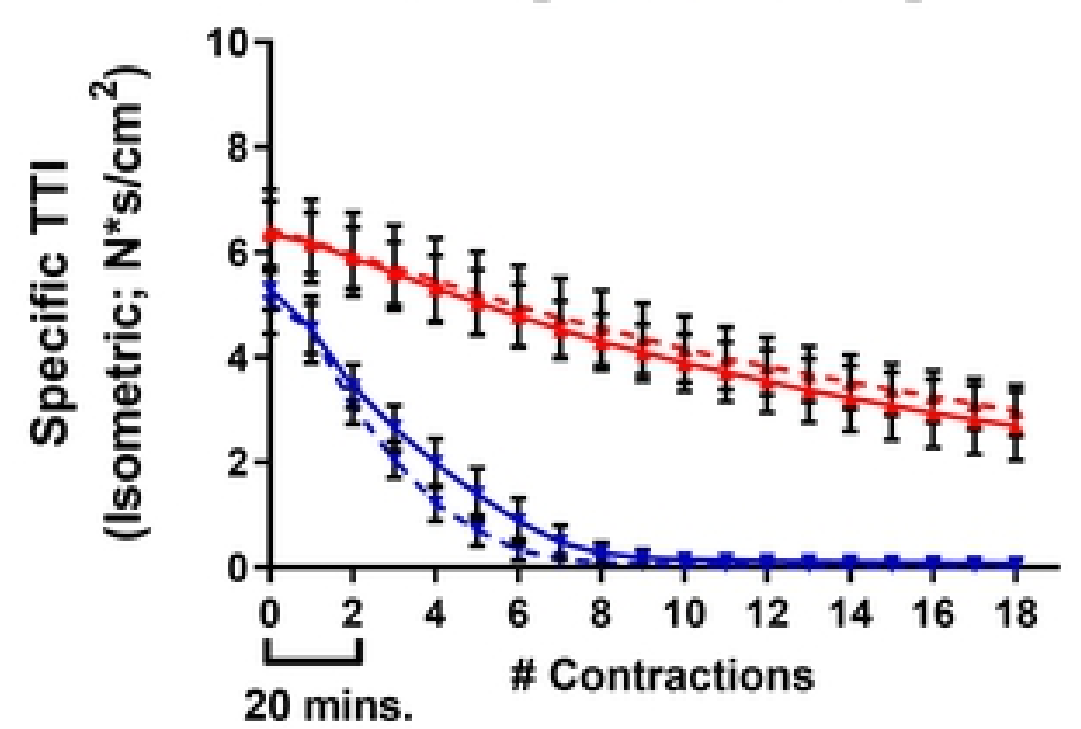

C

$$
\begin{aligned}
& \rightarrow \text { Fed } \mathrm{O}_{2} \quad \text {-.. Fasted } \mathrm{O}_{2} \\
& \rightarrow \text { Fed } \mathrm{N}_{2} \quad \text {-.. F Fasted } \mathrm{N}_{2}
\end{aligned}
$$

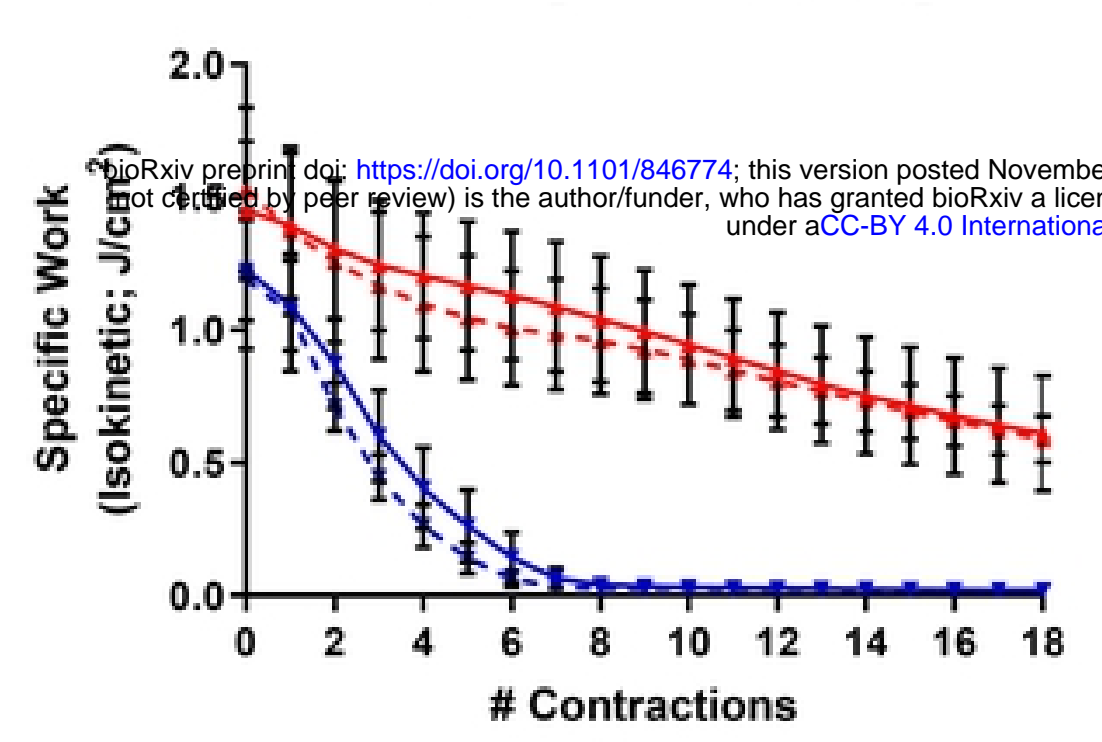

E
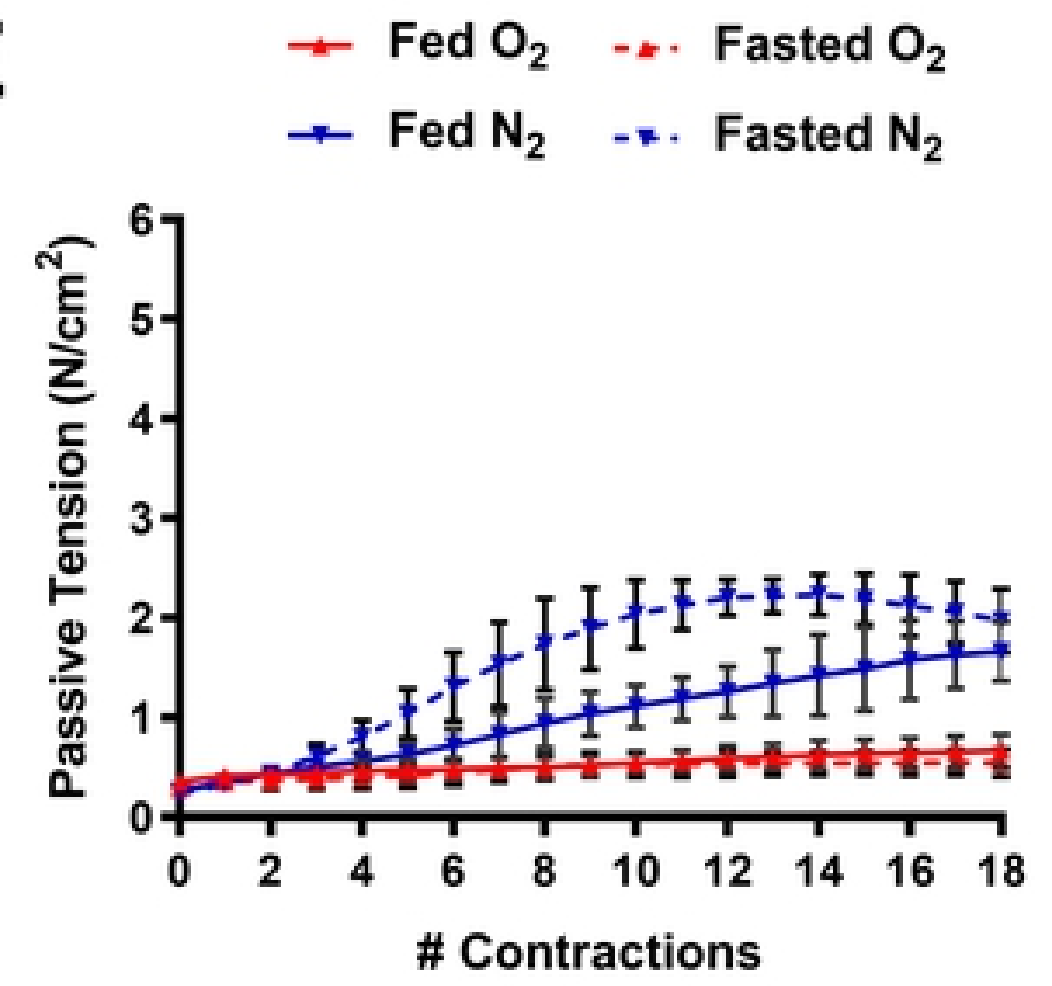

$\mathbf{G}$

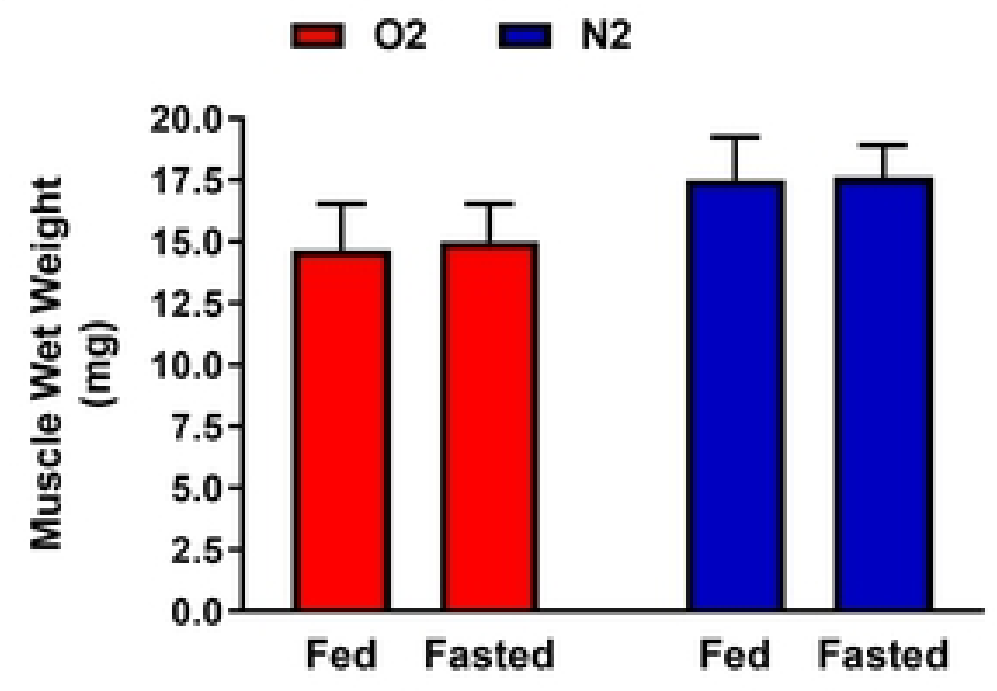

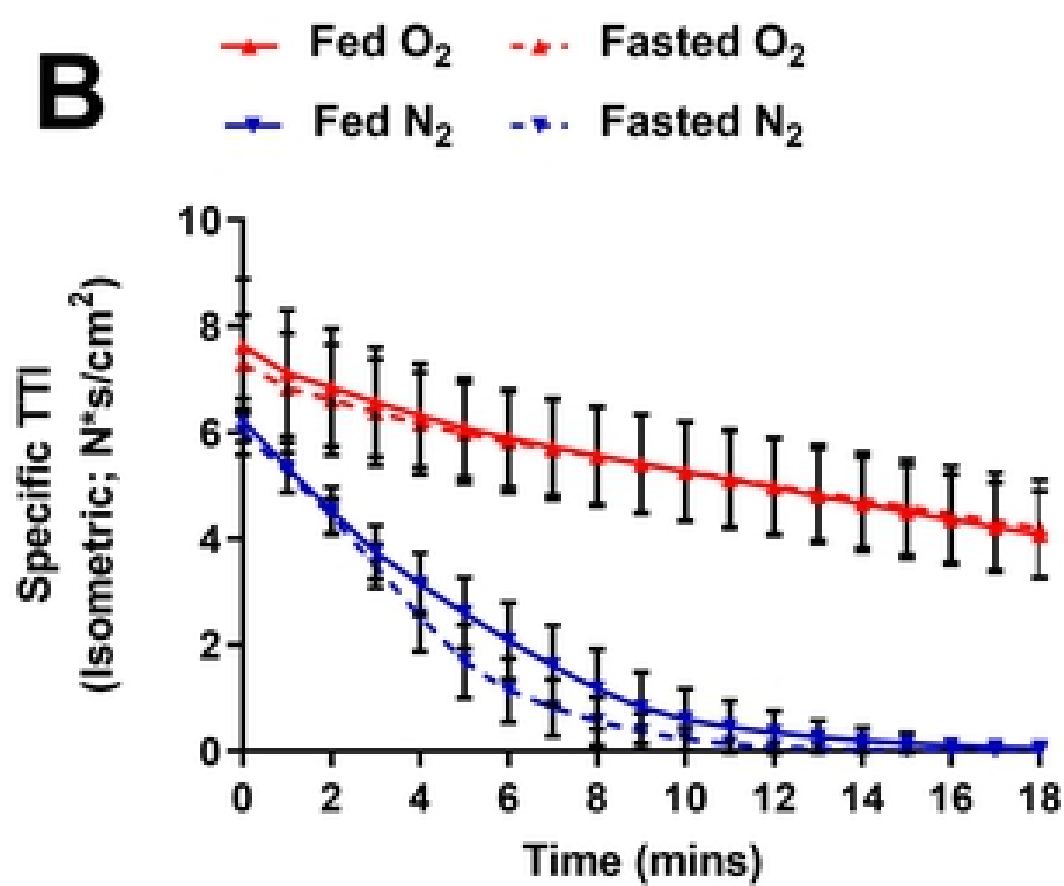

D
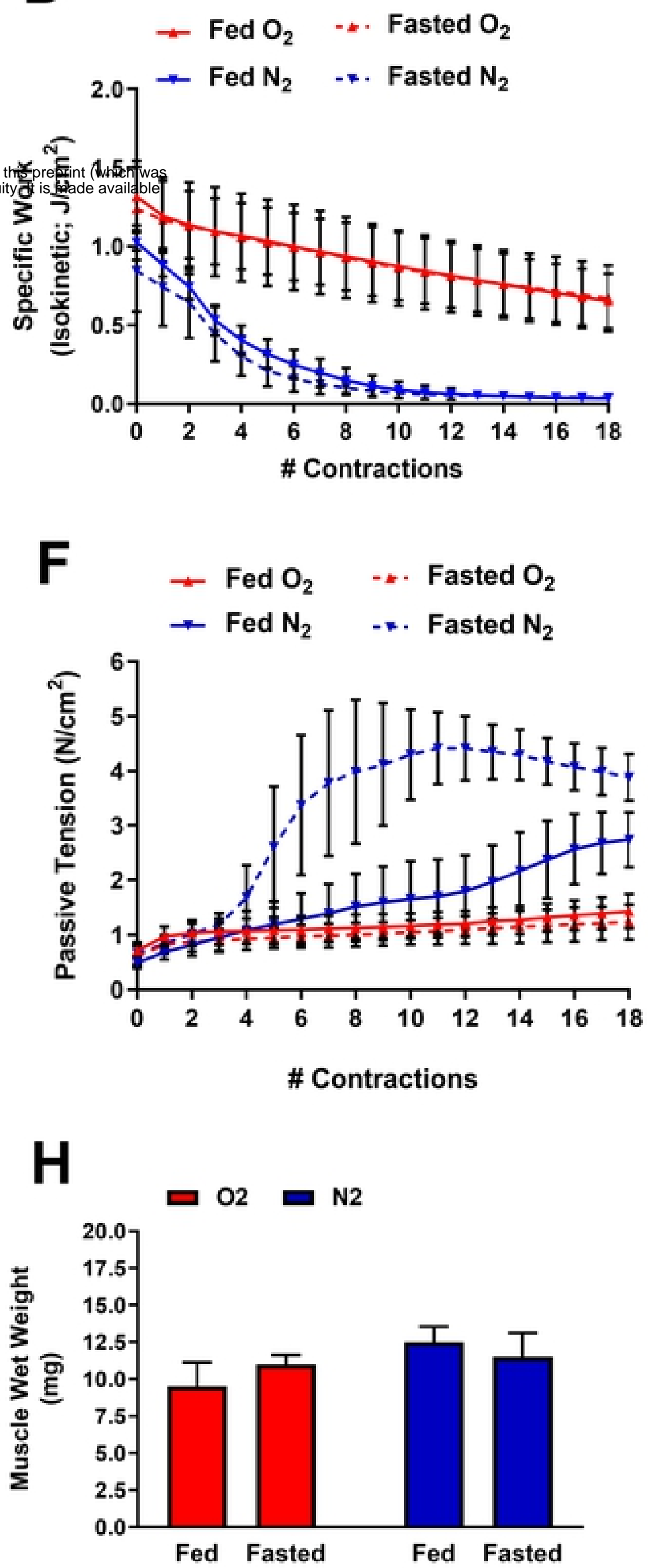

Figure 3 


\section{EDL}

A

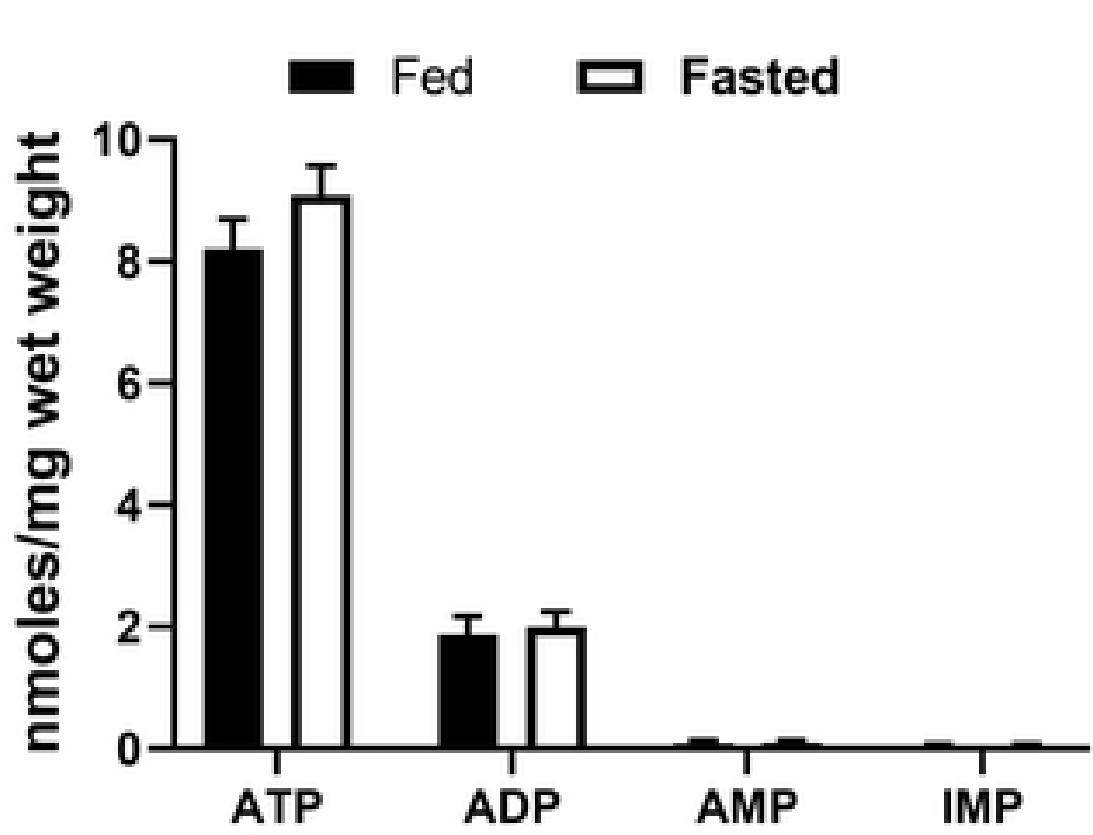

B

\section{Soleus}

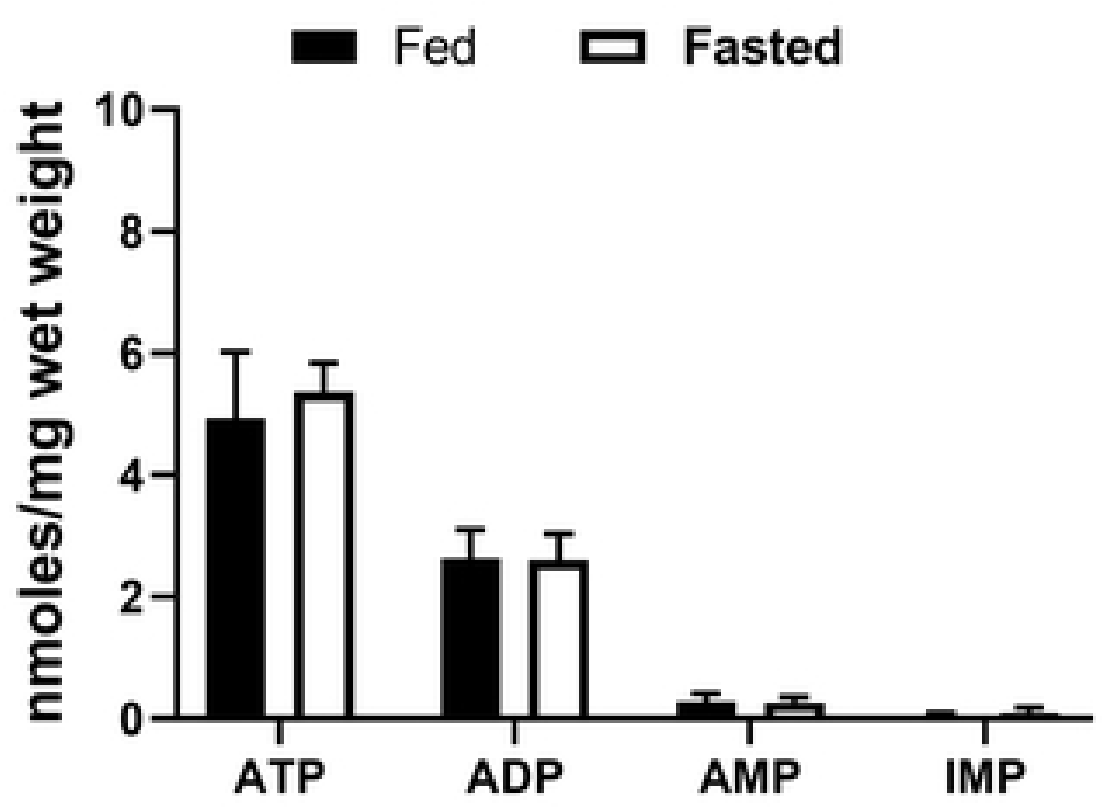

C

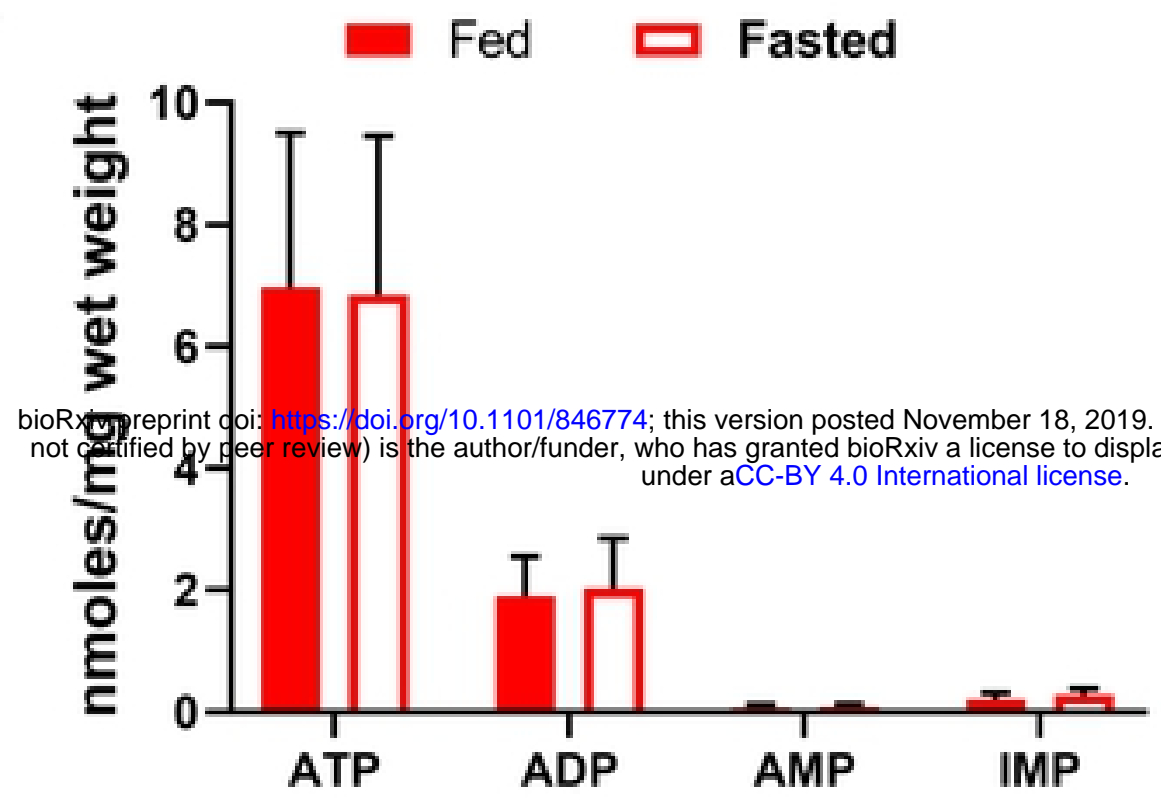

E

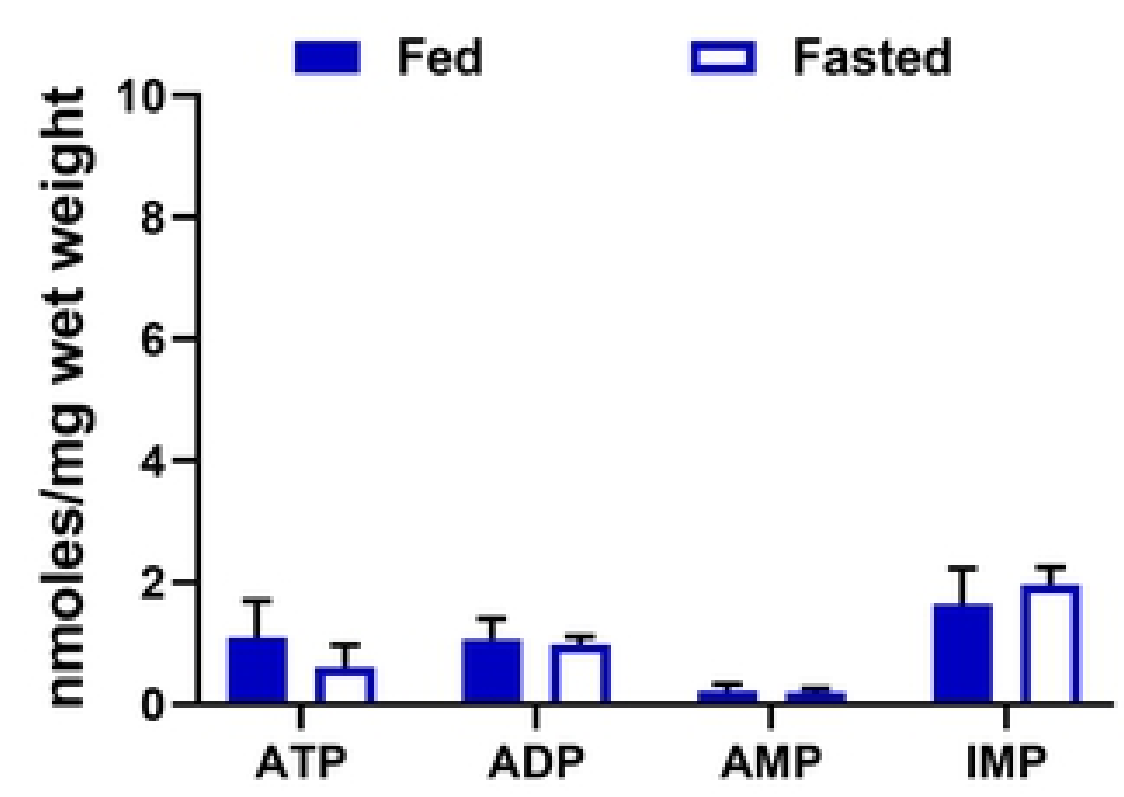

G
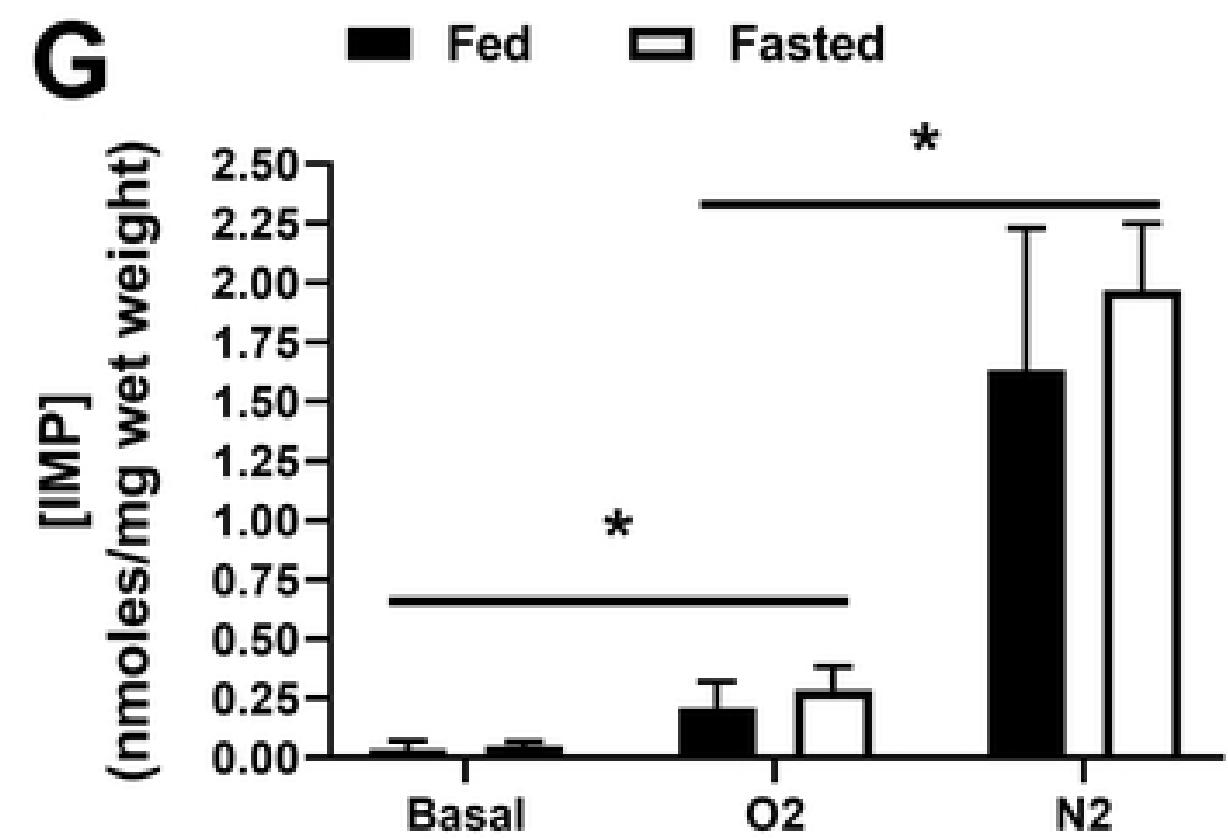

D

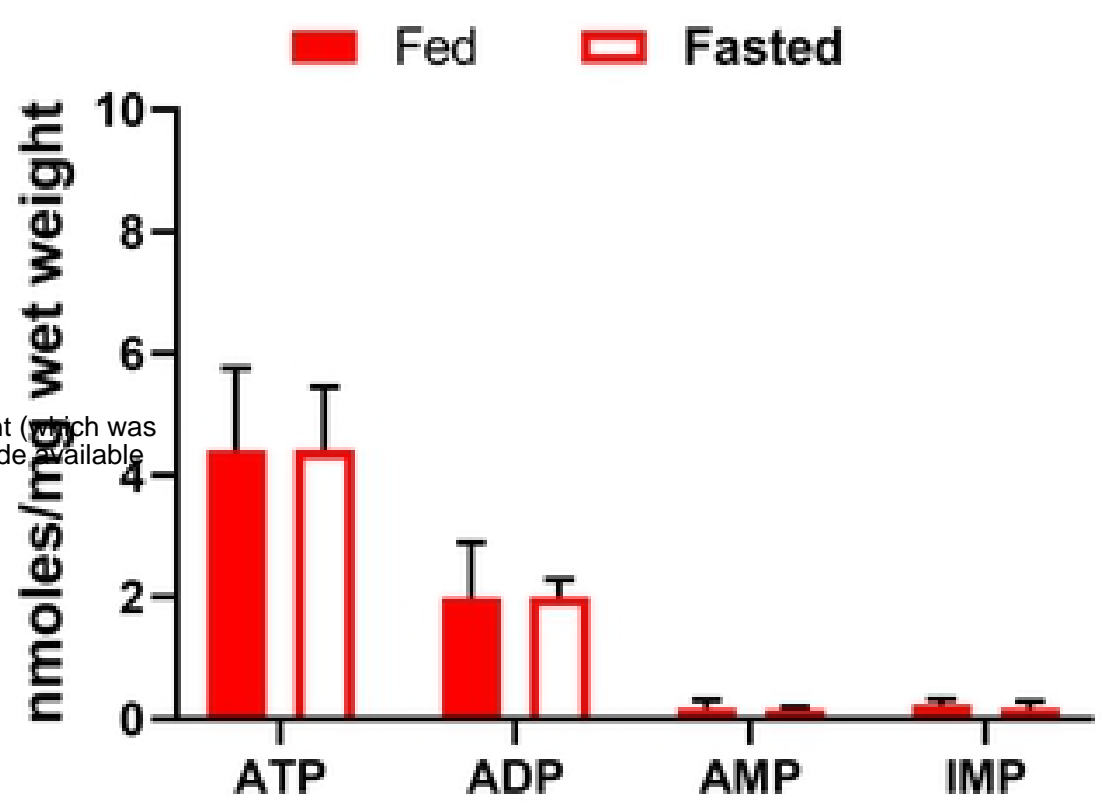

$\mathbf{F}$

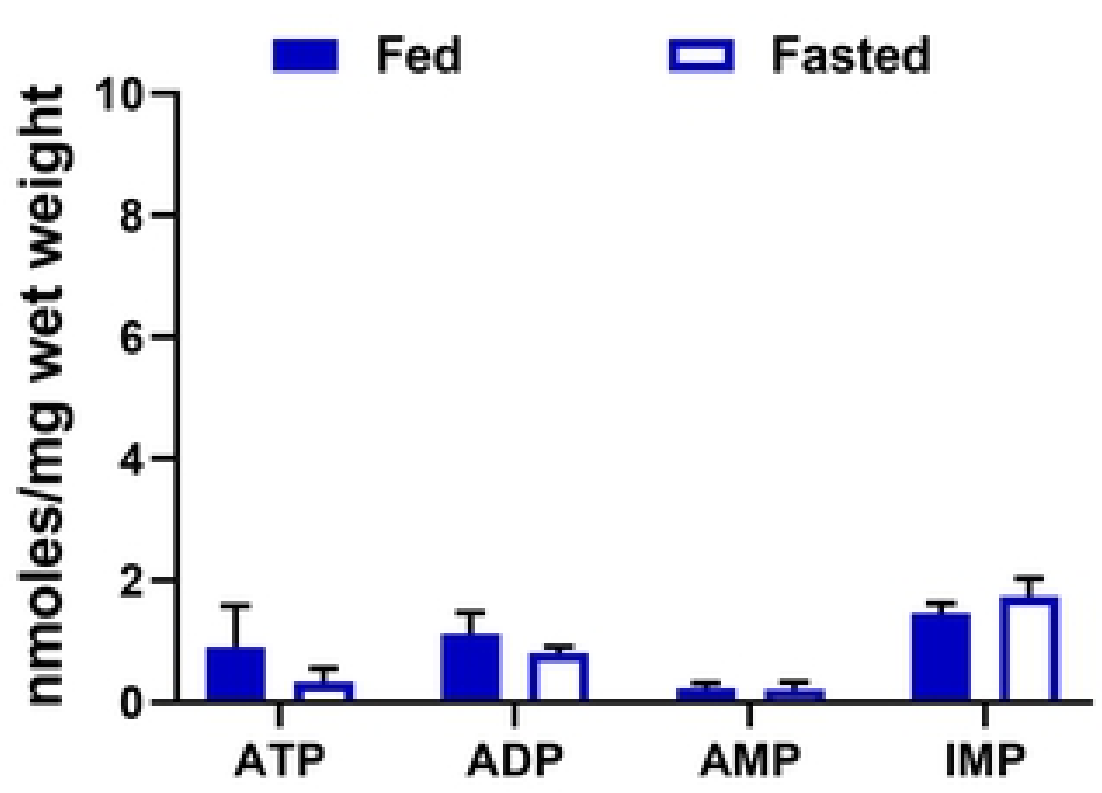

H

n Fed 口 Fasted

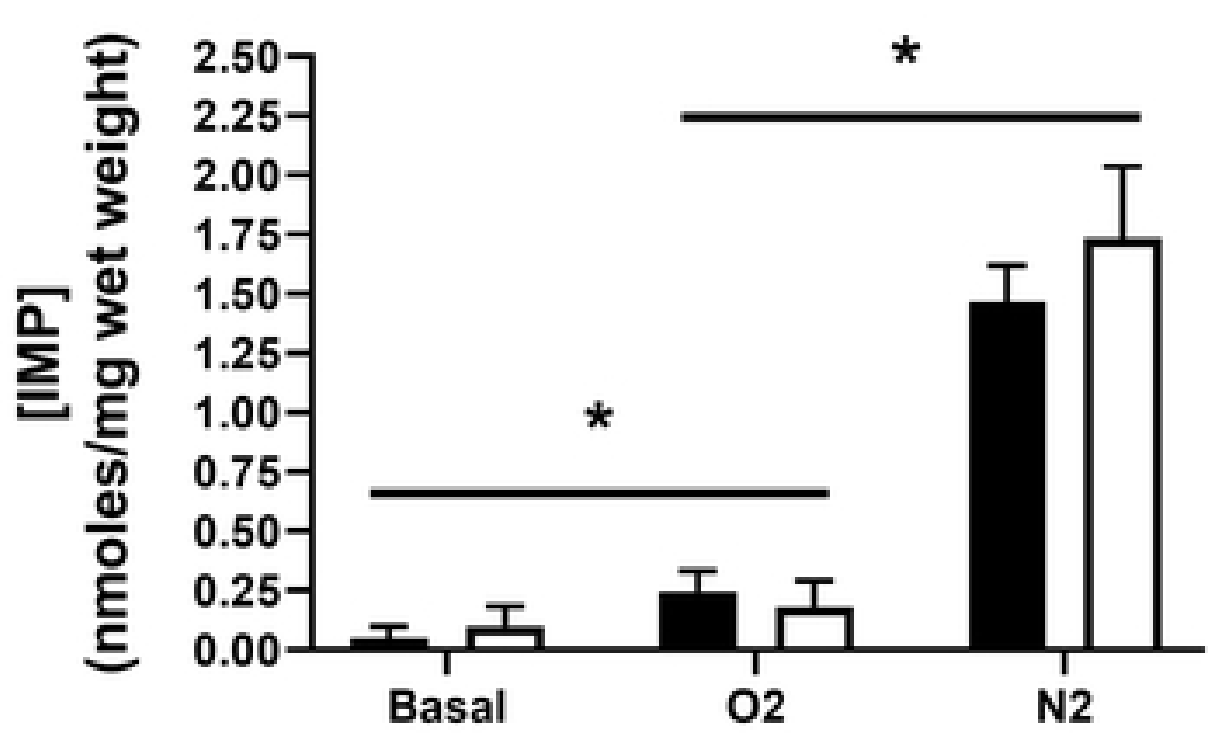

Figure 4 
